\title{
Liquid jet eruption from hollow relaxation
}

\author{
Élisabeth Ghabache ${ }^{1}$, Thomas Séon ${ }^{1} \dagger$ and Arnaud Antkowiak ${ }^{1}$ \\ ${ }^{1}$ Sorbonne Universités, UPMC Univ Paris 06, CNRS, UMR 7190 Institut Jean Le Rond d'Alembert, \\ F-75005 Paris, France
}

(Received 23 December 2013; revised 29 September 2014; accepted 28 October 2014)

\begin{abstract}
Using a model experiment, we explore the dynamics of inertial liquid jets arising from a gravitational cavity collapse. The focus of the study is to elucidate the link between both the dynamical and kinematical properties of the jet and the initial cavity geometry, for a wide range of physical parameters. We demonstrate that the jets exhibit shape similarity and reveal a robust relationship between the jet tip velocity and the initial cavity geometry, regardless of the details of the collapse process. We argue that this relation reflects a flow focusing mechanism, and we propose a simple model capturing the key features of the erupting jet velocity scaling. Finally, the relevance of these results to other jets occurring in e.g. large bubble detachment or wave impact on walls is discussed.
\end{abstract}

Key words: drops and bubbles, interfacial flows (free surface)

\section{Introduction}

Liquid jets or threads naturally occur in a variety of situations. The rupture of the fine liquid cap covering a small quiescent champagne or oceanic bubble preludes a fast chain of events including the fast recoil of the thin liquid film, the resorption of the cavity through emission of capillary waves, the ejection of a narrow and violent jet resulting from wave focusing and, ultimately, the rupture of this jet into a multitude of liquid fragments forming a spray (MacIntyre 1972). The billions of ink droplets needed to print a single page with drop-on-demand inkjet printheads also arise from the disruption of a liquid jet: the printheads feature piezoelectric actuators squeezing and pressurizing the ink chamber, resulting in the ejection of an ink jet rupturing into perfect equally-sized droplets (Dong, Carr \& Morris 2006). On a wider scale, when a shallow-water wave meets a steep cliff or harbour wall, a violent vertical jet can be thrown upwards without any actual impact on the wall (Peregrine 2003). This phenomenon, called flip-through, is responsible for the spectacular liquid projections observed from the oceanfront on windy days. These few examples illustrate some commonplace situations where a sudden overpressure is released through the development of a liquid jet. This overpressure may be of e.g. capillary, gravity or inertial origin and is often found to be mediated with a cavity collapse. Interestingly, the size distribution of the droplets resulting from the ultimate fragmentation of these different jets exhibit generic features (Villermaux 2007), but

$\dagger$ Email address for correspondence: thomas.seon@gmail.com 
(a)

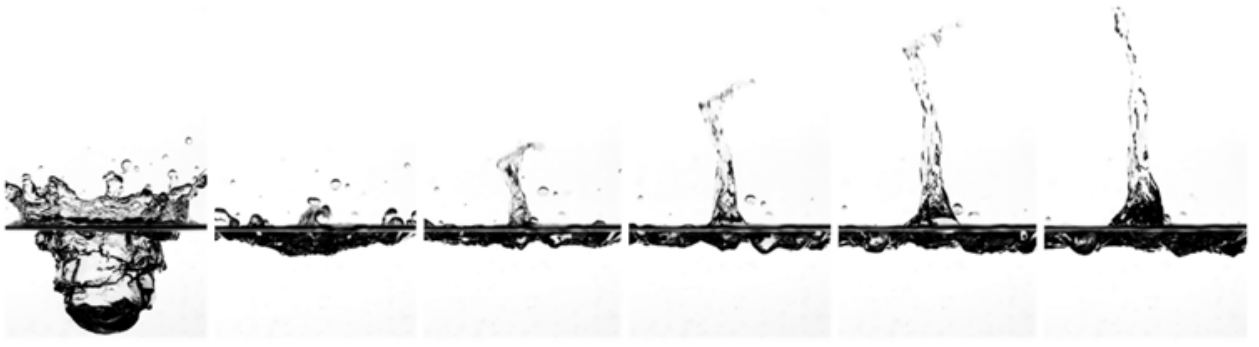

(b)

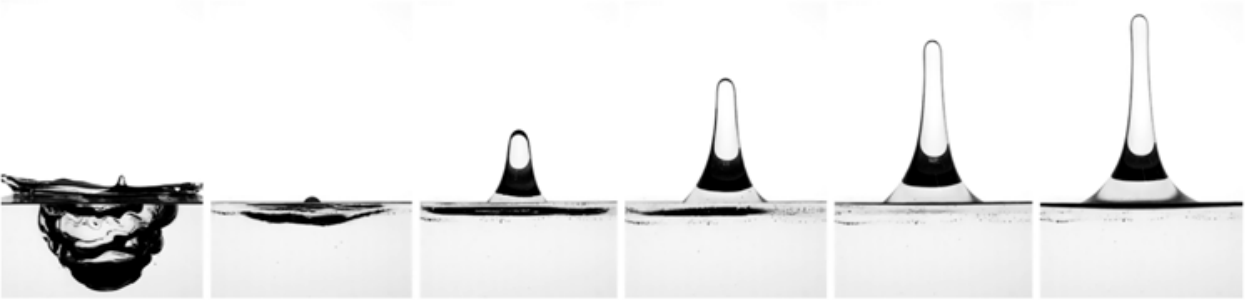

FIGURE 1. Time sequences of the jet induced by the relaxation of a centimetre-sized hollow in the free surface of two different liquids: $(a)$ water $(\mu=1 \mathrm{mPa} \mathrm{s})$ and $(b)$ a water-glycerol mixture of viscosity $\mu=833 \mathrm{mPa}$ s. Each sequence starts with a snapshot of the cavity, shaped by an air pulse, at its maximum depth $H$ and about to relax. On the second image the cavity is reversing giving rise to the high-speed jet observed on the following images. In $(a)$ the time lapse between the two first snapshots is $\Delta t_{1-2}=36 \mathrm{~ms}$ and between the following images is $\Delta t=7.5 \mathrm{~ms}$. The maximum hollow depth is $H=4.6 \mathrm{~cm}$, the width is $L=6.3 \mathrm{~cm}$ and the jet velocity is $V_{\text {tip }}=3.59 \mathrm{~m} \mathrm{~s}^{-1}$. In (b) $\Delta t_{1-2}=60 \mathrm{~ms}, \Delta t=27.5 \mathrm{~ms}, H=6 \mathrm{~cm}, L=10 \mathrm{~cm}$ and $V_{t i p}=1.85 \mathrm{~m} \mathrm{~s}^{-1}$.

the jet shape influence in the construction of these statistics remains unclear: do liquid jets exhibit generic characteristics as well?

A large body of literature is dedicated to the dynamics of cavities, starting with their formation (Duclaux et al. 2007; Bisighini et al. 2010) up to their collapse, which can involve a smooth cavity reversal (Cooker \& Peregrine 1990; Longuet-Higgins 2001; Antkowiak et al. 2007; Tagawa et al. 2012) or a finite-time hydrodynamic singularity (Zeff et al. 2000; Duchemin et al. 2002; Gekle et al. 2009). However much less work has been devoted to the description of the subsequent jet dynamics. Among relevant studies, Hogrefe et al. (1998) characterized the shape of various types of singularityrelated jets in terms of power laws; other groups have described jet tip velocities (Zeff et al. 2000; Duchemin et al. 2002), often in connection with singular collapse processes as well.

In this paper, we study the dynamics of an archetypical inertial liquid jet, arising from the non-singular collapse of a large cavity. The focus of the study is to elucidate the link between both dynamical and kinematical characteristics of the jet and the initial cavity geometry, for a wide range of physical parameters. To this end, we consider a model experiment where an initial cavity, shaped into a given form using a short air pulse, relaxes sparking a fast inertial liquid jet (see figure 1). After a description of the important stages in the jet development ( $\$ 2)$, we explore the dependence of the erupting jet velocity on the liquid properties and the cavity geometry. As a result we propose a robust scaling law for the jet velocity $(\$ 3)$. We further demonstrate that both the initial cavities and the outflowing jet exhibit shape similarity $(\S 4)$. We build on this observation and propose a simple model capturing 
the key features of the jet velocity dependence on the cavity shape. Finally, the relevance of these results to jets developing in different contexts is discussed in $\S 5$.

\section{Experimental description}

\subsection{Experimental set-up}

Our experiment consists of shaping a hollow in a free liquid surface by blowing a short air pulse from a nozzle: as soon as the airflow stops, the cavity relaxes under gravity to form a liquid jet. Both the depth $H$ and the width $L$ of the initial cavity can be tuned by adjusting appropriately the air pulse intensity and spreading: the higher the air pulse velocity, the deeper the cavity and the larger the height of the nozzle from the free surface, the wider the cavity. Typical heights and widths range from 3 to $10 \mathrm{~cm}$ and typical cavity aspect ratios $H / L$ from 0.25 to 1.1 (a hemispherical cavity would have an aspect ratio $H / L=0.5$ ). The liquid, contained in a transparent tank $(30 \mathrm{~cm} \times 30 \mathrm{~cm} \times 35 \mathrm{~cm})$, is a water-glycerol mixture. Depending on the proportions of water and glycerol, the liquid viscosity can be set in the range $\mu=1-1300 \mathrm{mPa}$ s. Note that temperature-induced variations have been accounted for throughout this study. The mixture density ranges from $\rho=1000$ to $1250 \mathrm{~kg} \mathrm{~m}^{-3}$. Pure ethanol of surface tension $\sigma=22.3 \mathrm{mN} \mathrm{m}^{-1}$ and viscosity $\mu=1.2 \mathrm{mPa}$ s has also been used in order to vary the surface tension. The hollow relaxation and jet dynamics are analysed through ultrafast imaging. Typically, images of the back-lit scene are obtained at 2000 f.p.s. using a digital high-speed camera (Photron SA-5).

\subsection{Qualitative overview of the jet dynamics}

Figure 1 illustrates two typical jetting events induced by the relaxation of a large surface hollow (whose width $L$ is greater than the capillary length). The sequence in figure $1(a)$ has been obtained with pure water as the working liquid while the one in figure 1(b) corresponds to a water-glycerol mixture 800 times more viscous. On the first images, the depression hollowed out in the liquid by the air pulse has reached its maximum depth $H$ and is about to relax. Just after maximal expansion, the liquid starts flowing in so as to fill the entire cavity. But this filling process is not just a simple relaxation to the minimal potential energy state (i.e. flat surface): during the collapse the cavity curvature reverses as a prelude to the eruption of a violent liquid jet (Lavrentiev \& Chabat 1980). It is noteworthy that the developing vertical jet can reach heights larger than ten times the initial depth $H$ of the cavity. This is a strong indication that the pressure built up during the collapse far exceeds the hydrostatic pressure: the gravity-driven flow focuses energy and momentum. Such focusing is generic in jet eruption and can be observed in situations as diverse as impact-driven liquid jets (Antkowiak et al. 2007), needle-less injection systems (Tagawa et al. 2012) or even granular jets (Lohse et al. 2004).

Insights into the role played by viscosity can readily be gained by comparing figures $1(a)$ and 1(b). Apart from its obvious smoothing action on the cavity, jet and lip surfaces, viscosity also proves to slow down the jet, as the time between each snapshot of figure 1 is much longer for the viscous case $\left(\Delta t_{\text {viscous }}=27.5 \mathrm{~ms}>\Delta t_{\text {water }}=7.5 \mathrm{~ms}\right)$. It is noteworthy that this slowing action of viscosity is achieved before jet eruption as evidenced by the jet velocity, which is seen to be constant in both sequences, at least before the final deceleration due to gravity.

A global overview of the jetting event is provided in figure 2 with a spatio-temporal diagram depicting the whole jet dynamics with the important stages in its chronology: 


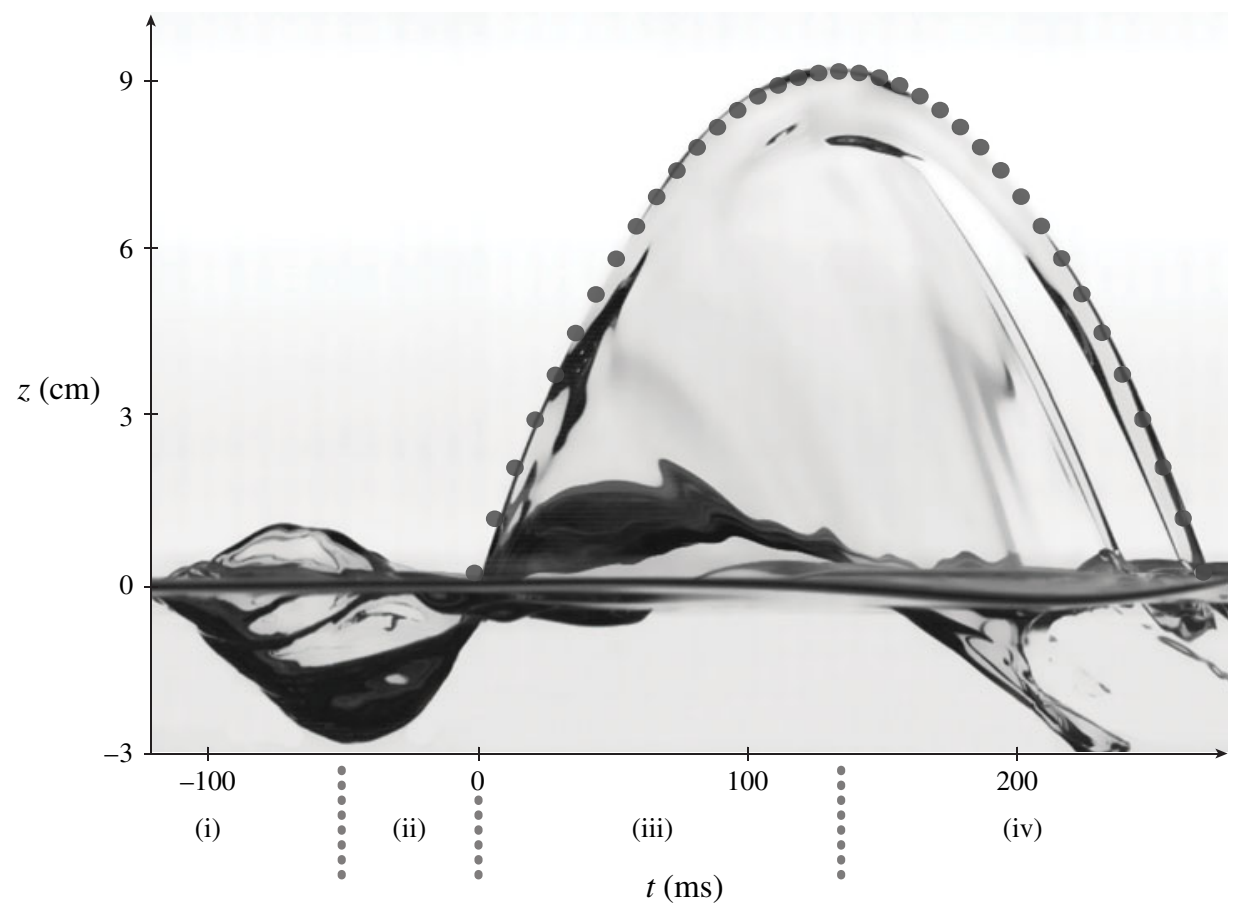

FIGURE 2. Spatio-temporal diagram presenting (i) the cavity formation, (ii) the cavity relaxation and reversal, (iii) the jet development and (iv) the jet collapse. These four steps are indicated in the timeline. The time origin $(t=0)$ is taken when the jet crosses the free surface $z=0$, and the local slope gives the jet velocity $V_{\text {tip }}$. The dotted curve represents the free-fall parabola corresponding to this jet eruption velocity $V_{\text {tip }}$. In this particular case the liquid viscosity is $\mu=10 \mathrm{mPa} \mathrm{s}$, the jet velocity is $V_{\text {tip }}=1.33 \mathrm{~m} \mathrm{~s}^{-1}$, the cavity maximum depth and width are $H=2.9 \mathrm{~cm}$ and $L=5.5 \mathrm{~cm}$.

(i) cavity formation up to maximum depth $H$, (ii) cavity relaxation and reversal, (iii) jet eruption and development and (iv) final jet collapse and/or disruption. From the curvature of the spatio-temporal trace it can readily be seen that the collapse phase involves an acceleration twice as important as gravity. Over-accelerations are typical of 'cumulative' phenomena (Lavrentiev \& Chabat 1980) and are commonly observed for example in wave impact events, where values as large as a thousand times that of gravity have been reported (Peregrine 2003). Just after eruption, the jet velocity $V_{\text {tip }}$, measured on the spatio-temporal diagram, is also seen to be important compared to the characteristic gravito-inertial velocity $\sqrt{g H}$ (here $V_{\text {tip }}=1.33 \mathrm{~m} \mathrm{~s}^{-1}>\sqrt{g H}=0.53 \mathrm{~m} \mathrm{~s}^{-1}$ ). Actually, in all the reported experiments, the Froude number $\mathrm{Fr}=V_{\text {tip }} / \sqrt{g H}$ was systematically observed to be greater than unity. Once above the free surface, the jet tip follows a ballistic trajectory characterized by a free-fall parabola (see dotted curve figure 2), even for liquids a hundred times more viscous than water (see the supplementary material available at http://dx.doi.org/10.1017/jfm.2014.629). This ballistic behaviour demonstrates that viscosity and surface tension barely affect the jet development. The purely inertial description assumption for the jet dynamics employed in this paper results from this key observation. 


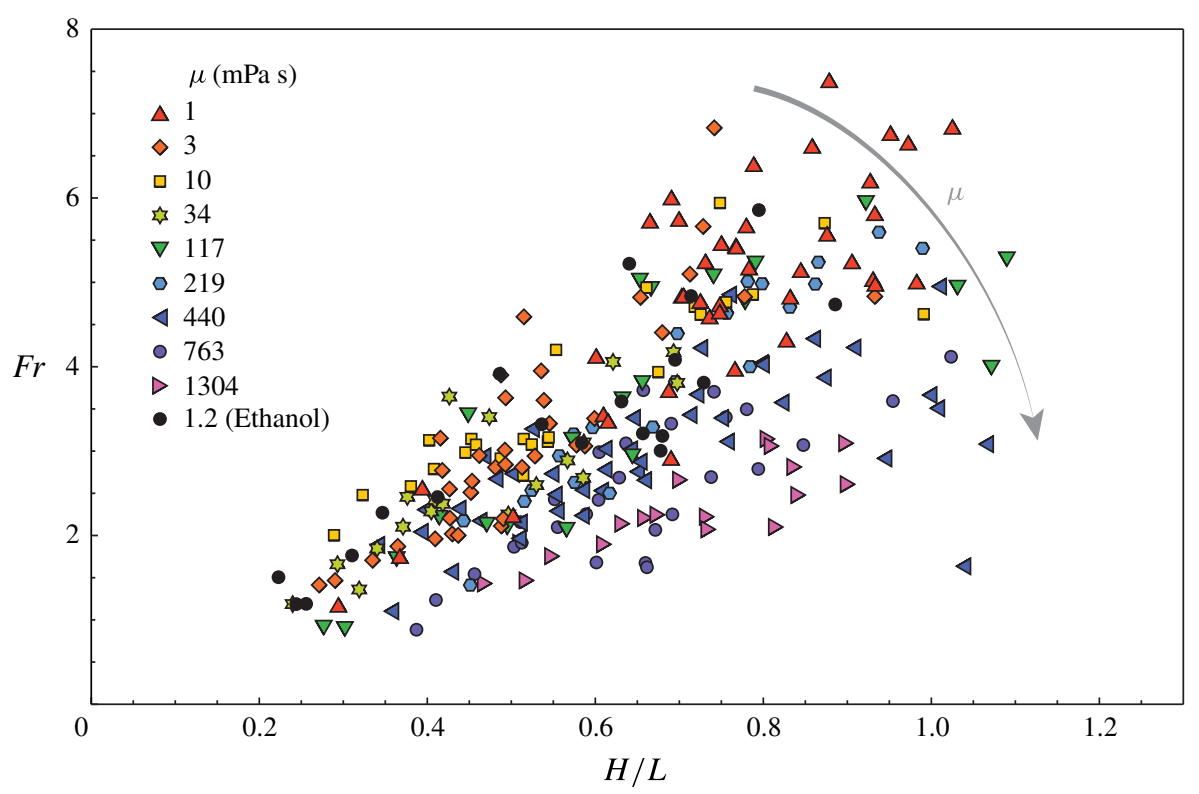

FIgURE 3. (Colour online) The Froude number $F r=V_{\text {tip }} / \sqrt{g H}$ increases with the aspect ratio $H / L$. The damping action of viscosity $\mu$ naturally lowers the eruption velocity (see arrow).

\section{Universal scaling for the jet eruption velocity}

We now investigate how the jet eruption velocity $V_{\text {tip }}$, completely determining the ballistic jet evolution, depends on the initial cavity geometry and on the liquid properties. We start by non-dimensionalizing the eruption velocity $V_{\text {tip }}$ with the natural gravito-inertial velocity scale $\sqrt{g H}$ and the depth $H$ with the width $L$. The resulting relation between the Froude number $F r=V_{\text {tip }} / \sqrt{g H}$ and the cavity aspect ratio $H / L$ is represented figure 3 for nine mixtures with viscosities distributed in the range $\mu=1-1300 \mathrm{mPa} \mathrm{s}$ and a pure ethanol solution of surface tension $\sigma=22.3 \mathrm{mN} \mathrm{m}^{-1}$ and viscosity $\mu=1.2 \mathrm{mPa}$ s. Two experimental facts directly emerge from the picture: first the eruption Froude number becomes larger with increasing cavity aspect ratio (i.e. with narrower cavities) and, second, the viscosity has a damping action on the jet velocity. While the latter observation is certainly to be expected, the dependence of the eruption Froude number on $H / L$ might be surprising. While studying liquid invasion into initially empty pipes, Lorenceau et al. (2002) reported that the liquid-column rise velocity was systematically found to be very close to $\sqrt{g H}$. In this 'rigid cavity filling' experiment, the Froude number therefore tends to one irrespective of the tube radius. The significant difference observed in velocity scaling in otherwise analogous experiments will later prove to be related to the energy and momentum focusing occurring in cavity relaxation.

Intending to obtain a robust scaling law for the eruption velocity also accounting for the action of viscosity and neglecting the capillary effects, we have identified so far five geometrical or dynamical variables governing the value of the jet tip velocity:

$$
V_{t i p}=F(\rho, \sqrt{g H}, \mu, H, L)
$$




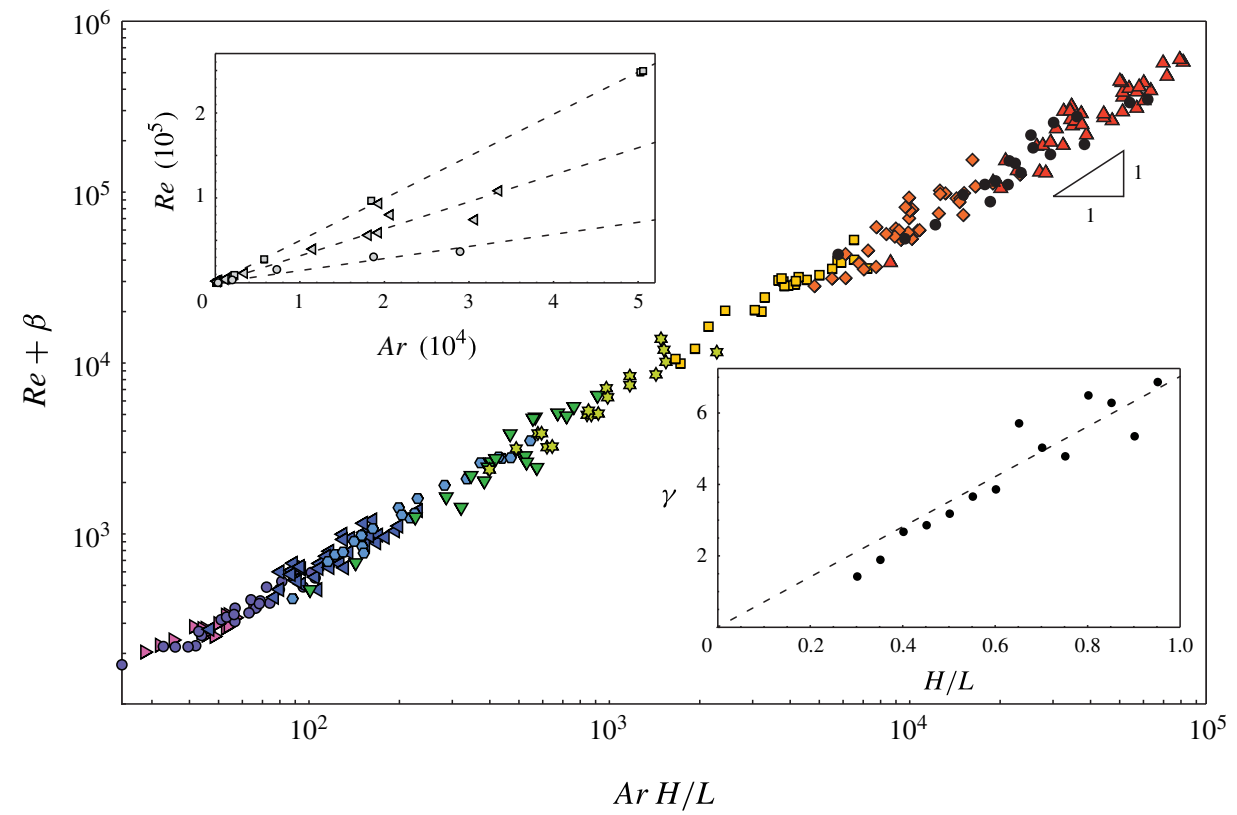

Figure 4. (Colour online) Experimental relation between $\operatorname{Re}+\beta$ and $A r H / L$ for the ten sets of experiments represented figure 3. Top left inset: Reynolds number $R e$ versus Archimedes number $A r$ for subsets of experiments having constant aspect ratio $H / L=0.3$, 0.5, 0.7. Each set of data is organized along a line of slope $\gamma(H / L)$ and all have the same $y$-intercept $\beta$. Bottom right inset: linear dependence of the slope $\gamma(H / L)$ on the cavity aspect ratio $H / L: \gamma(H / L)=\alpha H / L$. The constants $\alpha$ and $\beta$ defining the master curve are given by $\alpha=6.59$ and $\beta=104$.

Choosing $L, \rho$ and $\mu$ as independent scales, we use dimensional arguments (Barenblatt 2003) to recast the above equation into a relation between the dimensionless tip velocity $\operatorname{Re}=\rho V_{\text {tip }} L / \mu$, the Archimedes number $A r=\rho \sqrt{g H} L / \mu$ and the aspect ratio $H / L$ :

$$
R e=\mathscr{F}\left(A r, \frac{H}{L}\right) .
$$

We investigate experimentally the dependence of $R e$ on $A r$ by selecting subsets of experiments having constant cavity aspect ratio $H / L$. The relation between the Reynolds and Archimedes numbers is illustrated in the top left inset of figure 4 for three different $H / L$. It is instructive to note that each data subset is organized along a line of slope $\gamma$. While this slope appears to depend on $H / L$, detailed analysis of the data shows that each line presents the same $y$-intercept $\beta$, regardless of the aspect ratio value. As a result we may rewrite relation (3.2) as $\operatorname{Re}=\gamma(H / L) A r-\beta$. Further progress can be made by extracting the slope $\gamma(H / L)$ for a number of experimental subsets having various $H / L$, see bottom right inset of figure 4. Experimental data clearly demonstrate that the relation between $\gamma$ and $H / L$ is linear: $\gamma(H / L)=\alpha H / L$ with $\alpha$ a non-dimensional constant. This leads to the reformulation of (3.2) as the following scaling law:

$$
R e=\alpha \frac{H}{L} A r-\beta
$$


Figure 4 illustrates the experimental relation between $\operatorname{Re}+\beta$ and $A r H / L$ for the ten series of experiments conducted at various initial cavity geometries and different liquid properties. The collapse of all the experimental data on a single master curve of slope unity is remarkable and ranges over almost four decades, thereby confirming the relation between the jet eruption velocity and the initial hollow depth (3.3) and ruling out the effect of surface tension. The dimensional counterpart of this simple and robust scaling law can be expressed as

$$
V_{t i p}=\alpha \sqrt{g H} \frac{H}{L}-\beta \frac{v}{L},
$$

where $v=\mu / \rho$ denotes the liquid kinematic viscosity. Equation (3.4) makes it clear that the jet dynamics is merely a signature of the gravitational cavity collapse, but also that the offset $\beta$ in relation (3.3) has a viscous origin. We note that this relation also reflects a conversion of available energy (potential energy lessened by viscous dissipation) into jet kinetic energy. This point will further be developed in $\S 4.3$.

It is interesting to note that, unless experiments are performed with constant aspect ratio cavities, the tip velocity depends not only on the natural gravito-inertial scale $\sqrt{g H}$ but also on the global cavity geometry $(H / L)$. This is at variance with other systems involving a balance or a conversion of gravity potential energy into inertia, such as spherical-cap bubbles, gravity currents or rigid cavity filling (Lorenceau et al. 2002), where a local measure (typically of the front curvature radius or of the depth) allows the velocity to be inferred. In other words, a sequence of cavities having the same lateral extent $L$ but different depths $H$ will induce jets with velocities varying with $H^{3 / 2}$, as opposed to the previous examples where the dependence $H^{1 / 2}$ prevails. We relate the appearance of this unexpected exponent to an energy focusing phenomenon, as we show in the next section.

Though of inviscid nature, (3.4) indicates that the jet is strongly slowed down by viscous processes. As mentioned earlier $(\$ 2)$, in our experiment viscous damping occurs for the most part before jet eruption. We therefore expect dissipative phenomena to take place underneath the jet along with the strong deformation associated with the conversion of radial inflow into axial motion. This interpretation sheds light on the reason why viscosity does not appear in a prefactor but rather in an offset. Such a functional dependence for the dissipative action was already noted in Tagawa et al. (2012); there, the energy loss was associated with the heating of liquid by a laser beam.

It is, finally, worth noting that in the quite different context of a jetting event sparked off during large bubble formation, Seon \& Antkowiak (2012) did observe both the $H^{3 / 2}$ dependence of the jet velocity ( $H$ being a measure of the bubble height) and an analogous viscous offset, therefore putting in perspective the universality of the proposed scaling law.

\section{Shapes and dynamics: experimental rationalization and a simple model}

In the following we present a detailed description of the cavity and jet kinematics. In addition to improving our understanding of the cavity expansion and the jet dynamics, knowledge of the shapes, which are a signature of the cavity relaxation and the related flow focusing, will allow us to derive a simple energy balance model capturing the dependence of the jet velocity on the cavity geometry. 
(a)

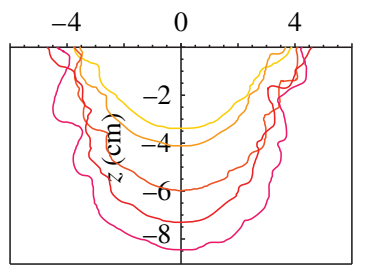

(b)

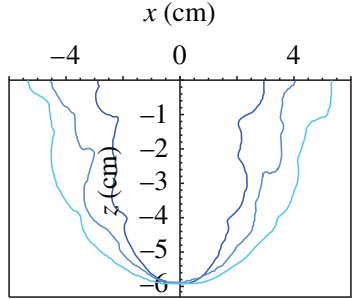

(c)

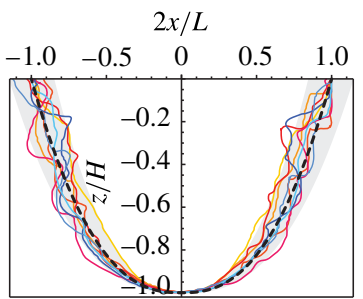

FIGURE 5. (Colour online) Different cavity profiles with $(a)$ almost the same width $(L)$ and different heights $(H)$ and a liquid viscosity of $\mu=219 \mathrm{mPa} \mathrm{s}$, and $(b)$ the same height, different widths and $\mu=440 \mathrm{mPa}$ s. (c) The same cavity profiles after rescaling: $z / H$ versus $2 x / L$. They all fall in the grey region: $z=x^{2.5 \pm 0.1}-1$ exhibiting a similar cavity shape. The black dashed line represent the curve $z=x^{2.5}-1$.

\subsection{Cavity shape similarity}

Blown cavities have been the focus of a number of studies in the past, partly driven by technological applications. To quote a few of these, we might mention vertical-take-off-and-landing craft that can experience loss of visibility or damage when landing over water or a soft terrain as a consequence of the soil erosion by a gaseous jet (Barton \& Edwards 1968), or the basic oxygen conversion process in the steel industry that makes use of a supersonic jet of oxygen impinging on molten iron to convert it into steel. Experiments modelling these phenomena have been reported for the last 50 years, with the notable contributions of Banks \& Chandrasekhara (1963) and Cheslak, Nicholls \& Sichel (1969). These studies have described how the geometrical properties of the cavity are linked to the blowing conditions (gas velocity, nozzle height and diameter). In particular, most of these works have considered steady cavity profiles to be reasonably well fitted with a parabola. Conversely, unsteady liquid cratering dynamics have been investigated mainly in the case of a point source, obtained for example by means of drop impact (Bisighini et al. 2010). In this type of experiment, the cavity shape is very close to an hemisphere and the dynamics of expansion-retraction is well described by the Rayleigh-Plesset equation. In our experiment, the pressure forces shaping the cavity are distributed along the cavity, i.e. not localized. (Curiously we have not been able to find a single study making use of impulsive blowing over a free surface. Stuhlman (1932) mentioned that Professor Foulk was using such a setup to produce jets, but the corresponding paper of Foulk only alluded to this experiment (Foulk 1932).)

Figure 5 reports cavity profiles at their deepest - that is, just before relaxation for eight different experiments corresponding to various values of the gas velocity (figure $5 a$ ) and of the nozzle height (figure $5 b$ ). We observe that by increasing the velocity of the air jet, deeper cavities can be formed keeping their width $L$ almost constant. Conversely moving the nozzle up allows wider cavities to be formed. It is notable that all these different cavities exhibit shape similarity. This is demonstrated figure $5(c)$ where the eight cavity profiles are plotted on the same graph after rescaling the horizontal coordinate with the cavity radius $L / 2$ and the vertical coordinate with the cavity depth $H$. All cavity contours fall onto a universal profile. The best fit for this master curve is given by $z / H=(2 x / L)^{2.5 \pm 0.1}-1$, with $z$ and $x$ the vertical and radial coordinates (dashed black curve in figure $5 c$ ). The shaded area represents the error bar taken on the exponent. 

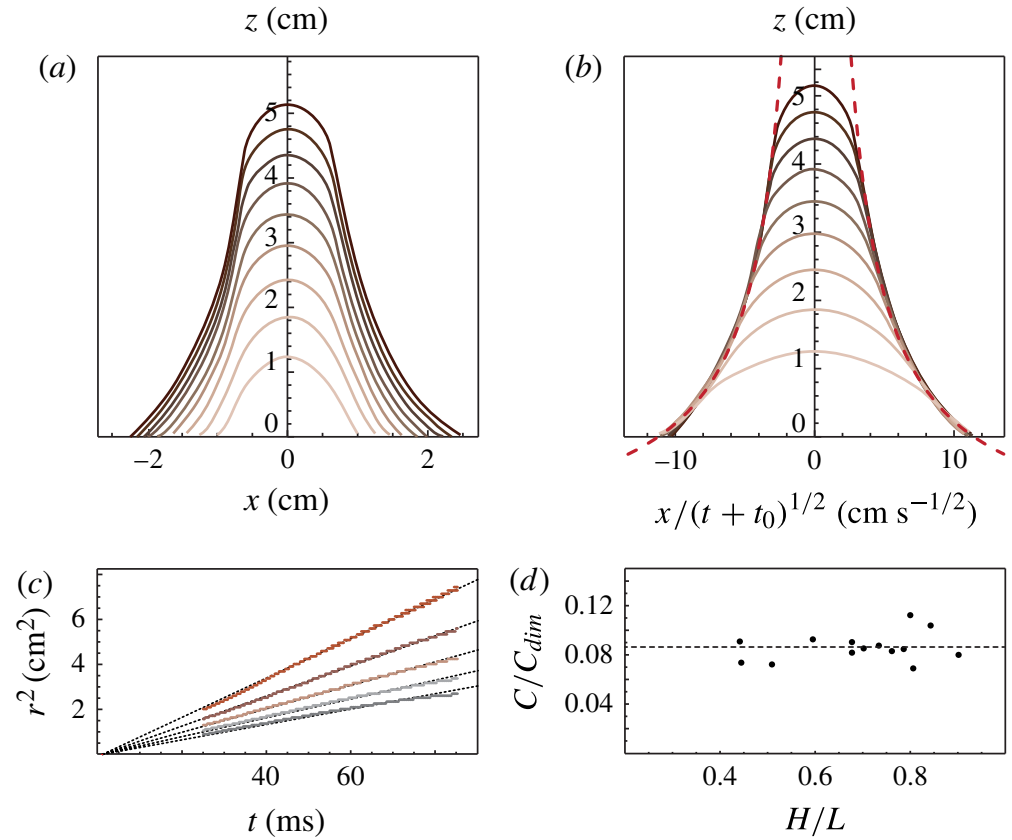

FIGURE 6. (Colour online) (a) Experimental jet profile evolution with time following cavity relaxation $\left(H=4.9 \mathrm{~cm}, L=9.6 \mathrm{~cm}, V_{\text {tip }}=1.41 \mathrm{~m} \mathrm{~s}^{-1}\right)$ in a liquid of viscosity $\mu=763 \mathrm{mPa}$ s. The interval between two profiles is $5 \mathrm{~ms}$. (b) The same jet profiles after rescaling of the $x$ coordinate by $\sqrt{t+t_{0}}$. The dashed curve follows the equation $z=C x^{-1}-z_{0}$. (c) Time dependence of the squared radius of the jet $\left(r^{2}\right)$ at different locations $z$ in the jet. $(d)$ Experimental relation between the jet radius prefactor $C$ non-dimensionalized by $C_{d i m}=(g H)^{1 / 4} L^{3 / 2}$ and the cavity aspect ratio $H / L$.

This process enables us to build similar hollows with various dimensions and aspect ratios. The further development of the jet only depends on this initial state, disregarding any prior cavity formation detail.

\subsection{Jet dynamics and shape similarity}

We now turn to the kinematics of jet formation, that starts with the cavity reversal. Figure 6(a) shows a typical sequence of successive jet profiles ( $\Delta t=5 \mathrm{~ms}$ between each) extracted from the same experiment. Here $z=0$ represents the far free surface. The roughly constant spacing between the successive jet tips is again an indication of the constant initial jet velocity (high Froude number eruption), consistent with the spatio-temporal diagram analysis (figure 2). The transverse time dependence of the jet is assessed by representing the evolution of the jet section $r_{j e t}^{2}(z, t)$ (disregarding the factor $\pi$ ) at five positions on the vertical axis $z$ (figure $6 c$ ). The linear trend curves capture satisfyingly the evolution of the jet section for every height, revealing that the jet radius varies as the square root of time. The experimental data therefore suggest the following functional form for $r_{j e t}(z, t)$ :

$$
r_{j e t}(z, t)=A(z) \sqrt{t+t_{0}}
$$


where the prefactor $A(z)$ is a function of the height $z$ only. This dependence of the jet shape on height is shown in the following to be completely constrained by the dynamics.

As mentioned earlier, experimental evidence suggests that the dynamics of the jet is barely affected by viscosity, capillarity or even gravity $(F r>1)$, at least for the range of parameters studied. Exploiting both the inertia-dominated character of the jet and its slenderness, we can formulate the governing equations expressing mass and momentum conservation in a one-dimensional setting (see Eggers (1997) for an historical account on one-dimensional approximations):

$$
\begin{gathered}
\frac{\partial r}{\partial t}+u \frac{\partial r}{\partial z}=-\frac{1}{2} r \frac{\partial u}{\partial z} \\
\frac{\partial u}{\partial t}+u \frac{\partial u}{\partial z}=0
\end{gathered}
$$

where $r(z, t)$ denotes the local jet radius and $u(z, t)$ the local velocity in a fluid slice.

For non-shock-forming feeding conditions, the velocity within the jet (i.e. the solution of $(4.2 b))$ is naturally attracted by the self-similar relaxation wave velocity profile:

$$
u(z, t)=\frac{z+z_{0}}{t+t_{0}},
$$

where $z_{0}$ and $t_{0}$ are space and time shifts (see appendix A). At the tip, the velocity field within the jet $u\left(z_{\text {tip }}\right)$ must match the tip velocity $V_{\text {tip }}$. This kinematic condition constrains $z_{0}$ and $t_{0}$ via the relation $V_{\text {tip }}=z_{0} / t_{0}$.

Analogously we now investigate jet shape similarity, by looking for solutions $r(z, t)$ of the mass conservation equation $(4.2 a)$ in the form of monomials $z^{a} t^{b}$. A one-parameter family of solutions such that $a=-(2 b+1) / 2$ is readily obtained. Using the linear time dependence of the jet section established experimentally, we infer that the particular exponent pair $(a, b)=\left(-1, \frac{1}{2}\right)$ is selected:

$$
r_{j e t}(z, t)=C \frac{\sqrt{t+t_{0}}}{z+z_{0}},
$$

where $C$ is a constant prefactor.

This result sheds light on the experimental jet profiles reported figure $6(a)$. Representing these profiles as a function of the self-similar variable $r / \sqrt{t+t_{0}}$ reveals that the jet actually develops in a self-similar sheath, see figure $6(b)$. It is noteworthy that the jet flanks adopt a perfect hyperbolic shape, consistent with the purely inertial prediction (4.4). The experimental observations reported figure 6 therefore demonstrate not only the shape similarity of the evolving jet (square-root-of-time spreading of the radius and hyperbolic shape), but also the validity of the assumptions made in obtaining (4.3) and (4.4). Interestingly Stuhlman (1932), performing an a posteriori analysis of prior experimental photographs obtained by Worthington, also found an hyperbolic shape for jets following a drop impact on a liquid surface.

Using the same natural scales for velocity $\sqrt{g H}$ and length $L$ as previously, we can non-dimensionalize the prefactor $C$ occurring in the expression for the radius (4.4). Without loss of generality, the resulting dimensionless number $C / C_{\operatorname{dim}}$ (where $\left.C_{d i m}=(g H)^{1 / 4} L^{3 / 2}\right)$ only depends on the cavity aspect ratio $H / L$. This dependence is tested experimentally (figure $6 d$ ) and it is found that $C / C_{d i m}$ is insensitive to the aspect ratio. From the experimental data we therefore conclude that the prefactor $C$ is approximately equal to $0.09(g H)^{1 / 4} L^{3 / 2}$. 


\subsection{Simple energy balance model}

Insights into the particular dependence of the jet velocity on the initial geometry of the cavity can be gained using first principles. First noting that the essence of jet formation is purely inviscid, we disregard in the following viscous effects (this hypothesis amounts to considering $\beta v \ll \alpha \sqrt{g H} H$ in (3.4)). The initial excess gravity potential energy associated with the initial hollow is

$$
U \sim \rho g L^{2} H^{2} .
$$

Note that we take no notice of the cavity-shape-dependent prefactor, nor of the kinetic energy of the remnant motions, in this elementary model as we are only interested in exponent determination. We seek in the following to express the conversion of this initial energy budget into jet kinetic energy. Recalling that gravity has a trivial effect on the jet dynamics (see $\$ 2$ ) we disregard such effects in the following for simplicity. The total kinetic energy of the flow can be decomposed into (i) the jet kinetic energy and (ii) the energy of the (typically dipolar) feeding flow pattern. As this last part decays with time, the asymptotic kinetic energy $T$ of the jet is the total energy of the flow. This quantity strongly depends not only on the velocity field within the jet, but also on the jet shape determined earlier. $T$ therefore can be written

$$
T=\int_{z=0}^{V_{t i p} t} \frac{1}{2} \rho u^{2}(z) \pi r_{j e t}^{2}(z) \mathrm{d} z \sim \rho C^{2} V_{t i p} \sim \rho \sqrt{g H} L^{3} V_{t i p} .
$$

Expressing the conversion of an $O(1)$ fraction of the initial potential energy $U$ into jet kinetic energy $T$ finally gives

$$
V_{t i p} \sim \sqrt{g H} \frac{H}{L} .
$$

On the other hand, in the tube filling experiment, the initial potential energy has the same expression (4.5) and the kinetic energy of the erupting liquid column is $T \sim$ $\rho V_{\text {tip }}^{2} L^{2} H$, leading to $V_{t i p} \sim \sqrt{g H}$, as observed in experiments.

\section{Conclusions and extensions}

Using a simple experiment, we have demonstrated experimentally that inertial jets resulting from a non-singular cavity collapse present robust characteristics for a wide range of parameters. In particular, jets were shown to exhibit both shape similarity and a clear dependence between the tip velocity and the initial cavity geometry, regardless of the details of the collapse process. The scaling relation between these quantities revealed the dependence of the velocity not only on the natural velocity scale $\sqrt{g H}$ but also on the cavity aspect ratio $H / L$. We pointed out that this dependence is a result of a flow focusing mechanism, whose action is embodied in the jet shape evolution equation (4.4) and reflected in the expression for the kinetic energy.

The scaling relation also exhibits an unusual viscous offset, suggesting that this viscous dissipation takes place in a localized region at the jet root. Above the feeding zone, the jet development is purely inertial as evidenced by the jet profile $r_{j e t}(z, t) \propto \sqrt{t} / z$ experimentally observed. We remark, though, that the present study is based on experimental measurements obtained shortly after jet emergence. Cumulative viscous effects - Trouton viscosity - become noticeable at later times for the highest viscosities. These viscous deviations are estimated in the supplementary 
material, where the maximal height reached by the jet is found to be in very close agreement with the theoretical ballistic height for two orders of magnitude in viscosity (1-100 $\mathrm{mPa} \mathrm{s})$.

We now discuss the relevance of these results from a broader perspective. Jets can be produced in a number of different ways. One of the most iconic may be drop impact on a deep pool, forming a crater quickly followed by the emergence of a vertical jet. Interestingly, upon analysing early photographs of drop impacts from Worthington, Stuhlman (1932) found an hyperbolic shape for the resulting jets, reminiscent of the hyperbolic profiles observed in this study. More recently, results from a series of drop impact experiments (Fedorchenko \& Wang 2004) or explosions at a water surface (Benusiglio, Quéré \& Clanet 2014) have revealed a linear relationship between the depth of the cavity and the maximum height of the jet. We infer from our results that the maximum jet height should scale with $H(H / L)^{2}$. Considering that, at maximum expansion, the cavity resulting from a drop impact or explosion is hemispherical (Fedorchenko \& Wang 2004; Bisighini et al. 2010; Benusiglio et al. 2014), i.e. has constant aspect ratio $H / L=0.5$, we see that the jet heights reported in these studies are consistent with our scaling relation. In the quite different context of large bubbles detaching from an underwater nozzle, we reported recently the formation of violent inertial jets developing inside the bubble just after disconnection (Seon \& Antkowiak 2012). Though the bubbling setup differs in many ways from the model experiment used in this study (large-scale convection pattern, collapsing flow, etc.), the jets initiated within the bubbles were found to follow an analogous scaling relation linking the jet velocity to the cavity geometry. The occurrence of a similar scaling relation in such different contexts therefore suggests some form of universality of our results. The question of the extent of the validity of the present results remains unclear. For example, in the context of wave impact, it has been noticed that the jet velocity could reach values as high as 20 times the natural velocity scale $\sqrt{g H}$ (Cooker \& Peregrine 1990), and that this prefactor was very sensitive to the precise shape of the wave (Longuet-Higgins 2001). Conversely in our experiments, we found our results to be very robust with respect to initial condition perturbations. Could it be possible to adapt our results to describe inertial jets in the flip-through phenomenon more robustly? Only more experimental data and theoretical developments will help answer this question, and identify the scope and generality of the present results.

\section{Acknowledgements}

L'Agence Nationale de la Recherche through its Grant 'DEFORMATION' ANR09-JCJC-0022-01, the Émergence(s) program of the Ville de Paris and the Direction Générale de l'Armement (DGA) are acknowledged for their financial support.

\section{Supplementary materials}

Supplementary materials are available at http://dx.doi.org/10.1017/jfm.2014.629.

Appendix A. The relaxation wave velocity profile as an inertial attractor (with Pierre-Yves Lagrée)

The pressureless and inviscid momentum equation within the liquid jet takes the form of Bürgers equation:

$$
\frac{\partial u}{\partial t}+u \frac{\partial u}{\partial z}=0
$$


We argue in the following that for any non-shock-forming injection conditions (that is, for any decreasing feeding velocity, as might be expected in this relaxation experiment), the velocity profile within the jet tends to the relaxation wave (or expansion fan) profile $z / t$.

Looking for a self-similar solution of (A 1), we search for monomial solutions $z^{\alpha} t^{\beta}$. The nonlinearity of the equation selects the single exponents pair $(\alpha, \beta)=(1,-1)$ which is precisely the relaxation wave profile $z / t$.

Now taking $\eta=z / t$ as the self-similar variable, we look for the behaviour of solutions $u(\eta, \tau)$ close to the self-similar profile (Eggers \& Fontelos 2009). Note that we have explicitly kept the time variable, now expressed via its $\operatorname{logarithm} \tau=\ln t$, to estimate departures from or convergence to the self-similar profile. Bürgers equation is then recast into:

$$
\frac{\partial u}{\partial \tau}=(\eta-u) \frac{\partial u}{\partial \eta} .
$$

Solutions close to the self-similar profile may be written as $u(\eta, \tau)=\eta+\epsilon u_{1}(\eta, \tau)$. Linearizing the equation around the self-similar state we end up with

$$
\frac{\partial u_{1}}{\partial \tau}=-u_{1}
$$

which admits an exponentially decaying solution in the logarithmic variable $\tau$. We conclude that the relaxation wave profile is a stable fixed point of the equation, and is therefore an inertial attractor for the jet dynamics.

\section{REFERENCES}

AntKowiak, A., Bremond, N., Le Dizès, S. \& VillermauX, E. 2007 Short-term dynamics of a density interface following an impact. J. Fluid Mech. 577, 241-250.

BANKS, R. B. \& CHANDRASEKHARA, D. V. 1963 Experimental investigation of the penetration of a high-velocity gas jet through a liquid surface. J. Fluid Mech. 15, 13-34.

Barenblatt, G. I. 2003 Scaling. Cambridge University Press.

BARTON, M. E. \& EdwARds, M. C. 1968 Model experiments of soil erosion by VTOL aircraft downwash impingement. J. Terramech. 5 (2), 45-51.

Benusiglio, A., Quéré, D. \& Clanet, C. 2014 Explosions at the water surface. J. Fluid Mech. 752, 123-139.

Bisighini, A., Cossali, G. E., Tropea, C. \& Roisman, I. V. 2010 Crater evolution after the impact of a drop onto a semi-infinite liquid target. Phys. Rev. E 82, 036319.

Cheslak, F. R., Nicholls, J. A. \& Sichel, M. 1969 Cavities formed on liquid surfaces by impinging gaseous jets. J. Fluid Mech. 36 (1), 55-63.

Cooker, M. J. \& Peregrine, D. H. 1990 Violent water motion at breaking-wave impact. Coastal Engineering Proceedings 1, 22.

Dong, H., CARr, W. W. \& Morris, J. F. 2006 Visualization of drop-on-demand inkjet: drop formation and deposition. Rev. Sci. Instrum. 77 (8), 085101.

Duchemin, L., Popinet, S., Josserand, C. \& Zaleski, S. 2002 Jet formation in bubbles bursting at a free surface. Phys. Fluids 14 (9), 3000-3008.

Duclaux, V., Caillé, F., Duez, C., Ybert, C., Bocquet, L. \& Clanet, C. 2007 Dynamics of transient cavities. J. Fluid Mech. 591, 1-19.

EgGers, J. 1997 Nonlinear dynamics and breakup of free-surface flows. Rev. Mod. Phys. 69 (3), $865-929$.

Eggers, J. \& Fontelos, M. A. 2009 The role of self-similarity in singularities of partial differential equations. Nonlinearity 22 (1), R1-R44. 
Fedorchenko, A. I. \& WANG, A.-B. 2004 On some common features of drop impact on liquid surfaces. Phys. Fluids 16 (5), 1349-1365.

FoulK, C. W. 1932 Foaming and priming of boiler water. Trans. Am. Soc. Mech. Eng. - Adv. Papers 54 (RP-54-5), 105-113.

Gekle, S., Gordillo, J.-M., Van Der Meer, D. \& Lohse, D. 2009 High-speed jet formation after solid object impact. Phys. Rev. Lett. 102 (3), 034502.

Hogrefe, J. E., Peffley, N. L., Goodridge, C. L., Shi, W. T., Hentschel, H. G. E. \& LAthrop, D. P. 1998 Power-law singularities in gravity-capillary waves. Physica D 123 (1-4), 183-205.

Lavrentiev, M. \& Chabat, B. 1980 Effets Hydrodynamiques et Modèles Mathématiques. Éditions MIR.

Lohse, D., Bergmann, R., Mikkelsen, R., Zeilstra, C., van Der Meer, D., Versluis, M., VAN DER WeEle, K., VAN DER Hoef, M. \& KUIPERS, H. 2004 Impact on soft sand: void collapse and jet formation. Phys. Rev. Lett. 93, 198003.

Longuet-Higgins, M. S. 2001 Vertical jets from standing waves. Proc. R. Soc. Lond. A 457 (2006), 495-510.

Lorenceau, E., Quere, D., Ollitrault, J.-Y. \& Clanet, C. 2002 Gravitational oscillations of a liquid column in a pipe. Phys. Fluids 14 (6), 1985-1992.

Macintyre, F. 1972 Flow patterns in breaking bubbles. J. Geophys. Res. 77 (27), 5211-5228.

Peregrine, D. H. 2003 Water-wave impact on walls. Annu. Rev. Fluid Mech. 35, 23-43.

Seon, T. \& Antkowiak, A. 2012 Large bubble rupture sparks fast liquid jet. Phys. Rev. Lett. 109, 014501 .

Stuhlman, O. JR. 1932 The mechanics of effervescence. Physics 2 (6), 457-466.

Tagawa, Y., Oudalov, N., Visser, C.-W., Peters, I.-R., Van Der Meer, D., Sun, C., Prosperetti, A. \& Lohse, D. 2012 Highly focused supersonic microjets. Phys. Rev. X 2, 031002 .

VillermauX, E. 2007 Fragmentation. Annu. Rev. Fluid Mech. 39 (1), 419-446.

Zeff, B. W., Kleber, B., Fineberg, J. \& Lathrop, D. P. 2000 Singularity dynamics in curvature collapse and jet eruption on a fluid surface. Nature 403 (6768), 401-404. 